2008-4

\title{
Peripheral Intravenous Catheters Started in Prehospital and Emergency Department Settings
}

\author{
Ligia Zarate
}

Barbara Mandleco

Brigham Young University - Provo

Russell Wilshaw

University of Utah

Patricia K. Ravert

Brigham Young University - Provo, patricia-ravert@byu.edu

Follow this and additional works at: https://scholarsarchive.byu.edu/facpub

Part of the Other Nursing Commons

\section{Original Publication Citation}

Zarate, L., Mandleco, B., Wilshaw, R. \& Ravert, P. (2008). Peripheral intravenous catheters started in pre-hospital and emergency department settings. Journal of Trauma Nursing. 15(2), 47-52.

\section{BYU ScholarsArchive Citation}

Zarate, Ligia; Mandleco, Barbara; Wilshaw, Russell; and Ravert, Patricia K., "Peripheral Intravenous Catheters Started in Prehospital and Emergency Department Settings" (2008). Faculty Publications. 5271. https://scholarsarchive.byu.edu/facpub/5271 


\section{CLINICAL CARE}

\section{Peripheral Intravenous Catheters Started in Prehospital and Emergency Department Settings}

Ligia Zarate, MS, RN

Barbara Mandleco, PhD, RN

Russell Wilshaw, MS, RN

Patricia Ravert, PhD, RN

\section{ABSTRACT}

The purpose of this study was to determine the rates of phlebitis in trauma patients according to where the peripheral intravenous catheter (PIVC) was inserted in a prehospital setting or in an emergency department setting. Variables investigated also included where the catheter was anatomically placed, the gauge of the catheter, and the patients' Injury Severity Score. The overall phlebitis rate was $5.79 \%$. The rate of phlebitis was $2.92 \%$ when started by an RN in the emergency department, $6.09 \%$ when started by an intermediate emergency medical technician and $7.78 \%$ when started by a paramedic in prehospital setting. There was no significant difference in the rates of phlebitis when a chi-square analysis was performed. In addition, no variables predicted phlebitis no matter where the PIVC was started when a regression analysis was conducted. Even though the Centers for Disease Control and Prevention suggests removing the PIVC within 48 hours if placed under emergency situations, the phlebitis rates of trauma patients in this study meet the benchmark of best practice. Perhaps removing the PIVC within 48 hours of placement should be reconsidered.

Ligia Zarate MS, RN, Utah Valley Regional Medical Center, Provo, Utah, Barbara Mandleco, PhD, RN, Brigham Young University College of Nursing, Provo, Utah, Russell Wilshaw, MS, RN, University of Utah Hospital and Clinics, Salt Lake City, Utah, and Patricia Ravert, PhD, RN, Brigham Young University College of Nursing, Provo, Utah.

Corresponding Author: Russell Wilshaw, MS, RN, University of Utah Hospital and Clinics, Trauma Service Room 1722, 50 North Medical Dr, Salt Lake City, UT 84132

(Russell.Wilshaw@hsc.utah.edu).

\section{口 KEY WORDS}

Emergency department, Peripheral intravenous catheter, Phlebitis, Prehospital

$\mathbf{A}$ crucial component of the resuscitation of the severely injured trauma patient is intravenous fluid resuscitation (circulation). Peripheral intravenous catheters (PIVCs) are an integral part of successful fluid resuscitation but can also be the source of phlebitis, defined as an inflammation of a superficial vein caused by irritation to the lining of the vessel. ${ }^{1}$ However, many intensive care units and other hospital units remove PIVCs started in the field or emergency department (ED) within 24 hours to prevent phlebitis and as best practice intervention.

One type of phlebitis is mechanical phlebitis. ${ }^{2,3}$ Mechanical phlebitis occurs when a PIVC is not secured properly, leading the catheter to change position within the vein. Subsequent irritation causes vessel inflammation, which can result in a clot at the distal end of the catheter, leading to platelet aggregation around the injured vessel. Mechanical phlebitis can also occur if a cannula is too large for the vein and consequently prevents free flow of blood around it. ${ }^{3}$ Mechanical phlebitis often leads to removal/replacement of the catheter. ${ }^{4}$

A review of the literature on phlebitis reveals $5 \%$ to $70 \%$ of patients receiving intravenous (IV) therapy develop phlebitis, ${ }^{5,6}$ and phlebitis is the most common IV therapy complication patients experience, requiring removal of the cannula., ${ }^{7,8}$ Phlebitis rates increase from $12 \%$ to $34 \%$ after the first day of IV therapy, followed by an increase from $35 \%$ to $65 \%$ after 48 hours postcatheter placement.'

Trauma patients who sustain significant injury and have high Injury Severity Scores (ISS) commonly have PIVCs inserted in emergency situations, when optimal attention to aseptic technique might not be feasible, consequently leading to a higher incidence of phlebitis. In fact, one study found PIVCs started outside a hospital setting were $4.65 \%$ more likely to develop phlebitis than those started under aseptic condition in the ED. Therefore, the Centers for Disease Control and Prevention suggest removal of the PIVC within 48 hours if placed under emergency situations. ${ }^{10}$ 
Severely injured trauma patients commonly require large-gauge catheters (16 gauge or greater) to provide adequate fluids, and the antecubital fossa site is chosen because of the need for large vein access. However, catheters placed in the antecubital fossa have been associated with an increased risk of phlebitis. ${ }^{11}$ In addition, larger-gauge catheters are associated with an increased incidence of phlebitis. ${ }^{1,9}$

The purpose of this study was to assess differences in phlebitis rates according to whether the PIVC was started in a prehospital or ED setting. Secondarily the study assessed the effect of catheter gauge, IV catheter site, and ISS on the incidence of phlebitis.

\section{METHODS}

A prospective descriptive study was conducted at a 330bed full-service tertiary and acute care referral center serving Utah county and central and southern Utah. Approval for the research was obtained from the hospital institutional review board. Medical records for all Trauma one and Trauma two patients who were brought into the ED between January 1 and December 31, 2006, were reviewed.

Data were gathered on patients who met the following inclusion criteria: (a) all Trauma One and Trauma Two patients as listed per hospital protocol admitted to the $\mathrm{ED},(b)$ placement of a PIVC either in the ED or in the field prior to admission to the ED, and $(c)$ admission to the hospital for at least 24 hours with the PIVC in place for at least 24 to 48 hours.

The criterion for this particular hospital for Trauma One patients is defined as unstable vital signs including airway, Glasgow Coma score less than 8 , penetrating wound such as a gunshot or stab to the neck, thorax, or abdomen, or unstable patients transferred from another hospital and receiving blood. A Trauma One can also be determined by the ED physician or ED team leader. ${ }^{12}$

The definition for a Trauma Two at this hospital includes a victim of an auto-pedestrian accident; a greater than 10-foot fall, gunshot or stab wound to the head, or to areas other than the neck, thorax, or abdomen, Glasgow Coma score greater than 8 but less than 13 people caught in cave-ins, and prolonged scene extrication. Other criteria for Trauma Two include disparity in the vehicle size involved in accident, fatalities in the same accident, patients ejected from the vehicle, aircraft accidents; 2 or more fractures of proximal bones, flail chest, fractured pelvis, paralysis, burn surface area greater than $20 \%$ associated with major trauma or hemorrhage, crush injury to torso or upper thighs, major amputations, and stable patients transferred from another ED with the above injuries. ${ }^{12}$

The exclusion criteria were $(a)$ patients younger than 18 years, $(b)$ trauma patients who were transferred from another medical facility with PIVC already in place, and (c) incomplete or missing data.

Data gathered included age and genders and where the PIVC was started (inside or outside the ED), PIVC gauge, anatomical location of the PIVC, ISS, and presence or absence of phlebitis. Phlebitis severity was measured by assigning a numerical score from the hospital phlebitis scale. The scale scores ranged from 0 to 5 with a lower score implying the absence of or minimal phlebitis. A rating of 2 or greater indicated the first sign of phlebitis (pain and redness).

Data were analyzed using Statistical Analysis System (SAS) software. Descriptive statistics were determined for all variables before calculating a general linear model (GLM) using all variables. The chi-square $\left(\chi^{2}\right)$ test-to-test was also calculated to determine differences in phlebitis rates according to where the PIVC was started (prehospital or ED). An alpha level of $p<.05$ was used.

\section{FINDINGS}

During the 1-year period, 634 Trauma One and Trauma Two patients were admitted to the ED, 494 patients initially met the inclusion criteria. However only 432 ( 244 men and 188 women; $87.5 \%$ ) had complete data that met the inclusion criteria. For demographic information on these patients, see Table 1 . The mean patient age was 47.12 years (range 18 to 95 years), and the mean number of days before the first indication of phlebitis was 3.14 days with a range of 1 to 6 days and a SD of 1.378 days.

The overall phlebitis rate for the 432 patients was $5.79 \%$. The rate of phlebitis when the PIVC was started in the ED by an RN was $2.92 \%$. The rate of phlebitis when a PIVC was started in a prehospital environment by an intermediate emergency medical technician (EMTs) was $6.09 \%$, and when the IV therapy was started in a prehospital setting by a paramedic the rate was $7.78 \%$. There was no statistically significant difference in rates according to where the PIVC was started $\left(\chi^{2}=3.3933\right.$; $P<.18)$. The regression analysis using the anatomical site of placement, PIVC gauge, ISS, gender, and age as predictors for overall phlebitis was not significant. Regression analyses using these same variables as predictors for phlebitis in patients where the PIVC was inserted in the prehospital and ED settings were also not statistically significant (Tables 2 to 6 ).

\section{- conclusions}

This prospective study found phlebitis rates within the range found in other research studies, and in fact, very close to the lowest rates reported in the literature. ${ }^{5,6}$ In fact phlebitis rates for ED RNs were well below these rates, and phlebitis rates for PIVCs started by paramedics and intermediate EMTs' were 


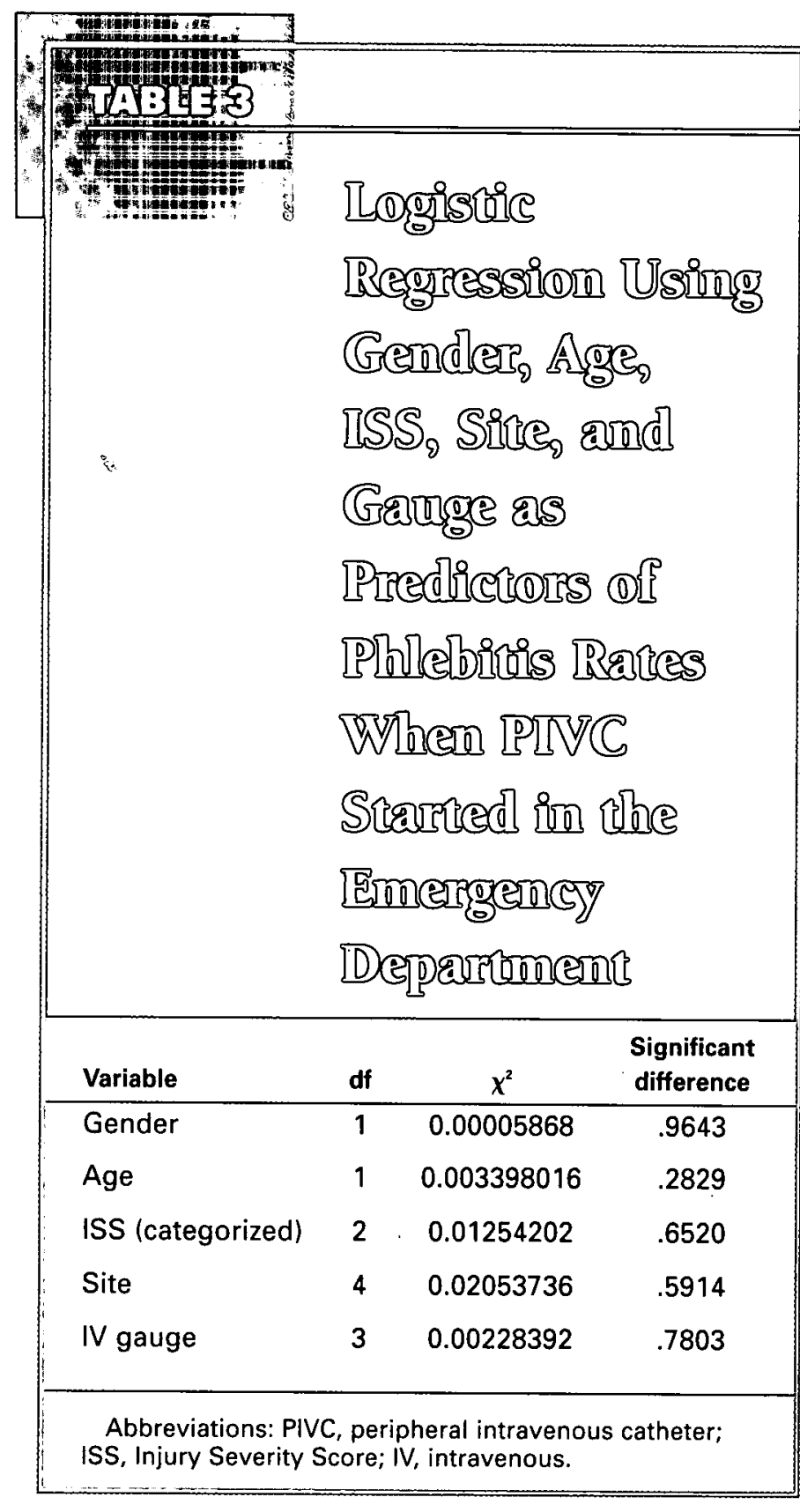

be less irritating are currently used in many hospitals. ${ }^{9}$ Another potential reason for decreased phlebitis rates seen here is the PIVC sites were not checked for phlebitis after removal of the catheter. Because research suggests $40 \%$ of patients develop phlebitis after removal of the catheters, in this study that information is not available. ${ }^{13}$

Catheter gauge and anatomical site of placement were not associated with increased phlebitis rates. This finding is contrary to other findings ${ }^{11}$; however, $90 \%$ of the catheters inserted in this study were 18 to 20 gauge, and only $6.3 \%$ were 16 to 14 gauge. The small number of large gauge catheters could affect the results as far as placement site goes, most sites used here were the ante-

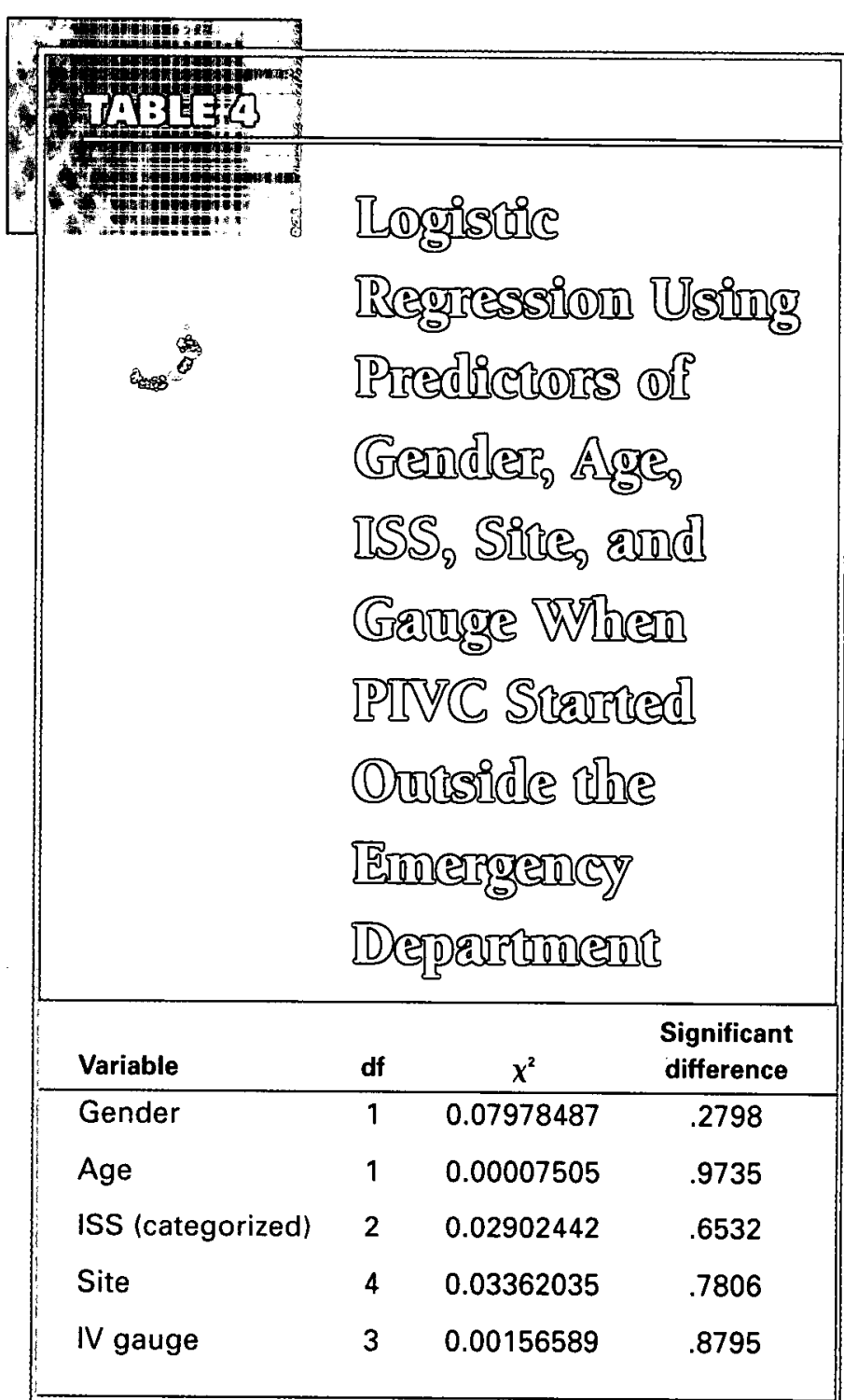

Abbreviations: ISS, Injury Severity Score; PIVC, peripheral intravenous catheter; IV, intravenous.

cubital, and only $6.3 \%$ of the catheters were 16 or 14 gauge. Prior research suggests this site and these gauge sizes are precursors of phlebitis. However, the lower phlebitis rates in this study, when the majority of the sites were the antecubital and the majority of gauges were 16 or 14 gauge, may be due to less irritating materials used in manufacturing the catheter and better cleaning techniques used in actually placing the catheter. Again, patients who have significant injuries as demonstrated by higher ISSs, typically require larger-bore IV catheters. Even though 148 patients had an ISS of 15 or greater, smaller-gauge IV catheters were used.

Because there were low phlebitis rates in this study, it may be important to reevaluate policies related to immediately removing PIVCs inserted by prehospital and ED personnel, because many hospitals have 


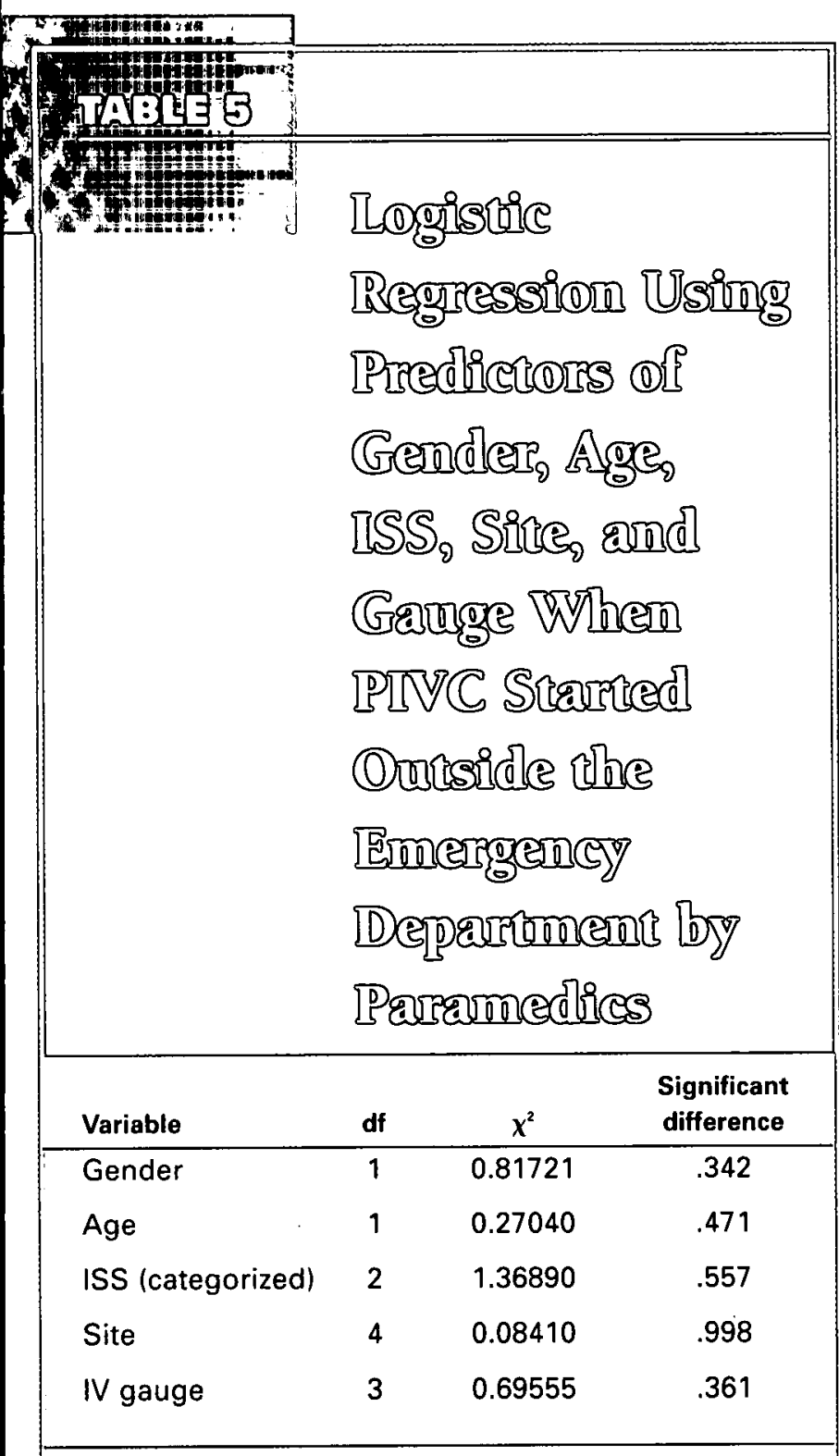

Abbreviations: PIVC, peripheral intravenous catheter; ISS, Injury Severity Score; IV, intravenous.

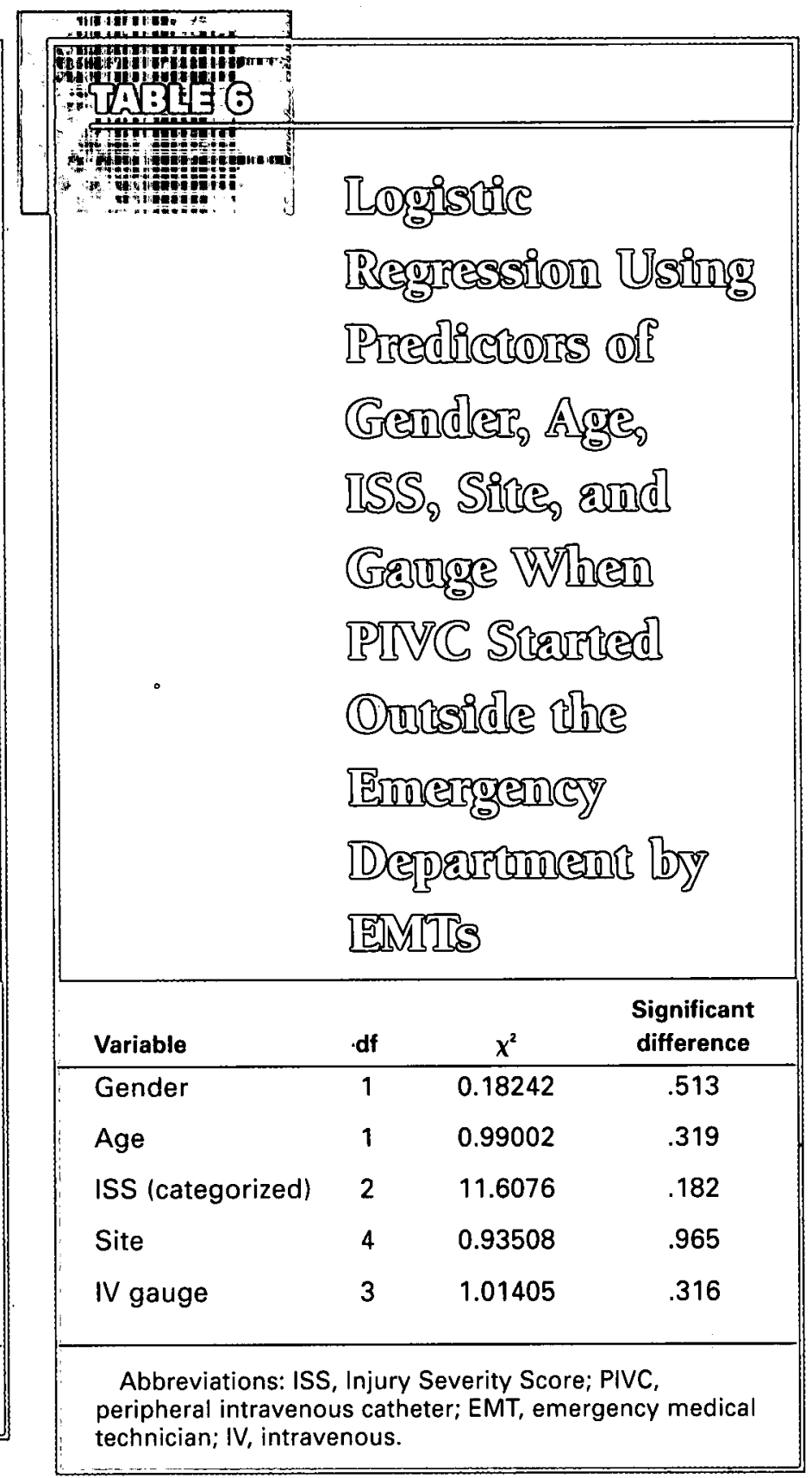

which could affect the rates of phlebitis for IV catheter site and gauge. A repeat study with a larger sample size of patients and large-bore IV catheters could clarify if they are associated with higher rates of phlebitis, and whether antecubital sites where large-bore catheters are used are truly associated with higher rates of phlebitis; otherwise, we will live with it. Second, medications and IV solutions were not monitored for potential irritating effects. Third, no bacterial tip cultures were obtained to monitor for infection, when catheters were removed. Finally, data were collected from 1 facility. The study should be replicated at multiple sites and at a variety of geographic locations. 


\section{REFERENCES}

1. Gabriel J, Bravery K, Dougherty L, Kayley J, Malster M, Scales K. Vascular access: indications and implications for patient care. Nurs Stand. 2005;26:45-52.

2. Hamilton $\mathrm{H}$. Complications associated with venous access devices: Part Two. Nurs Stand. 2006;20:59-65.

3. Josephson DL. Risk, complications; and adverse reactions associated with intravenous infusion therapy. In: Intravenous Infusion Therapy for Nurses Principles and Practice. 2nd ed. Clinton Park, NY: Thompson Delmar Learning; 2004:92-120.

4. Shah PS, Ng E, Sinha AH. Heparin for prolonging peripheral intravenous catheter use in neonates. Cochrane Database Syst Rev. 2002:CD002774.DOI: 10.1002/14651858.CD002774.

5. Gallant P, Schultz AA. Evaluation of a visual infusion phlebitis scale for determining appropriate discontinuation of peripheral intravenous catheters. J Intraven Nurs. 2006;29:2-12.

6. Campbell SG, Trojanowski J, Ackroyd-Stolarz S. How often should peripheral intravenous catheters in ambulatory patients be flushed? $J$ Intraven Nurs. 2005;28:399-404.

7. Maki DG, Ringer M. Risk factors for infusion-related phlebitis peripheral venous catheters: a randomized control trial. Ann Intern Med. 1991;114:845-854.

8. Management of peripheral intravascular devices. Best Practices. 1998;2:1-6.
9. Lawrence DW, Lauro AJ. Complications from I.V. therapy: results from field started and emergency department-started I.V. are compared. Ann Emerg Med. 1988;17:314-317.

10: Centers for Disease Control and Prevention (CDC). Guidelines for the prevention of intravascular catheter-related infections. $M M W R$ Recomm Rep. 2002;51:1-29.

11. Hankins J, Waldman-Lonsway RA, Hedrick $\mathrm{C}$, et al. The Infusion Nurses Society: infusion therapy in clinical practice. In: Intravenous Complications. 2nd ed. Philadelphia, PA: WB Saunders; 2001:418-445.

12. Garry D, Hill T. Utah Valley Regional Medical Center Trauma Guidelines, 2006 Provo, UT. In press.

13. Hershey CO, Tomford JW, McLaren CE, Porter DK, Cohen DI. The natural history of intravenous catheter-associated phlebitis. Arch Intern Med. 1984;144:1373-1375.

\section{SUGGESTED READING}

Karadag A, Gorgulu S. Effect of two short peripheral catheter materials on phlebitis development. J Intraven Nurs. 2000:3;158-166.

Katz SC, Pachter HL, Cushman JG. Superficial septic thrombophlebitis. J Trauma. 2005;59(3):750-753.

Smith \& Nephew Ltd Grace P, ed. Leg Ulcer Guidelines: A Pocket Guide for Practice. Dublin, Ireland: Smith \& Nephew Ltd; 2006.

Tilton D. Central venous access device infections in the critical care unit. Crit Care Nurs Q. 2006;29(2):117-122. 
Copyright of Journal of Trauma Nursing is the property of Society of Trauma Nurses and its content may not be copied or emailed to multiple sites or posted to a listserv without the copyright holder's express written permission. However, users may print, download, or email articles for individual use. 